\title{
Delphi Survey Round 2
}

\section{Indicators of Endemicity}

1. Which of the following indicators are relevant to classify endemicity of leprosy at sub-national level? Rank them in their order of importance: 1 being the most important and onward numbers being less important; two or more indicators may be ranked the same if they are equally important. Any indicator which you may consider non-relevant could be ranked as $N A .(\mathrm{N}=18)$

\begin{tabular}{|c|c|c|c|c|c|c|c|c|c|c|c|c|}
\hline \multirow[t]{2}{*}{ Indicator } & \multicolumn{10}{|c|}{ Ranking } & \multirow{2}{*}{$\begin{array}{l}\text { Rank } \\
\text { score } \\
(0-5) \\
\end{array}$} & \multirow{2}{*}{$\begin{array}{l}\% \\
\text { Rank } \\
\text { top-3 }\end{array}$} \\
\hline & 1 & 2 & 3 & 4 & 5 & 6 & 7 & 8 & 9 & $10+$ & & \\
\hline New case detection (number and/or rate) & 11 & 4 & 1 & & & & & & & & 4.1 & 88.9 \\
\hline New cases detected among children (number and/or rate) & 2 & 3 & 3 & 2 & 1 & & 1 & & & & 2.0 & 44.4 \\
\hline $\begin{array}{l}\text { Number of new and old cases found by survey and active } \\
\text { screening }\end{array}$ & 1 & & & & & & & & & & 0.3 & 5.6 \\
\hline New case trend & 1 & 1 & 1 & 1 & & & 1 & & & 2 & 0.8 & 16.7 \\
\hline Rate of skin smear positive cases per 100,000 population & 1 & & 1 & & & 1 & & & 1 & 2 & 0.4 & 11.1 \\
\hline Age-specific incidence rate & 1 & & 1 & & & & 1 & 1 & 1 & 1 & 0.4 & 11.1 \\
\hline Suspect cases (at the clinic) & 1 & & & & & 1 & & & 2 & 1 & 0.3 & 5.6 \\
\hline Proportion of G2D cases among total new cases detected & & 4 & 1 & 1 & & 2 & 1 & 1 & & & 1.2 & 27.8 \\
\hline Proportion of child cases among total new cases detected & & 2 & 1 & 5 & 1 & 1 & & & & & 1.2 & 16.7 \\
\hline New cases detected with G2D (number and/or rate) & & 1 & 3 & & 3 & & 1 & & & & 0.9 & 22.2 \\
\hline $\begin{array}{l}\text { Proportion of household contacts with leprosy during } \\
\text { routine monitoring }\end{array}$ & & 1 & & 1 & 1 & & 1 & 1 & & 2 & 0.4 & 5.6 \\
\hline $\begin{array}{l}\text { Number of new and old cases found by survey and active } \\
\text { screening }\end{array}$ & & & 1 & 2 & 1 & & & & & 2 & 0.4 & 5.6 \\
\hline Proportion of MB cases among total new cases detected & & & 1 & 1 & 3 & 2 & & 2 & & 1 & 0.4 & 5.6 \\
\hline Prevalence (number and/or rate) & & & & & 1 & 1 & & & & 2 & 0.1 & 0.0 \\
\hline Quality of control program (in terms of interventions) & & & & & & & & 1 & & 3 & 0.0 & 0.0 \\
\hline
\end{tabular}

\section{Usage of indicators to Determine Endemicity}

Please indicate to what extent you agree with the following statements:

2. In order to determine leprosy endemicity, we should use the indicator value of a single year. $(\mathrm{N}=18)$

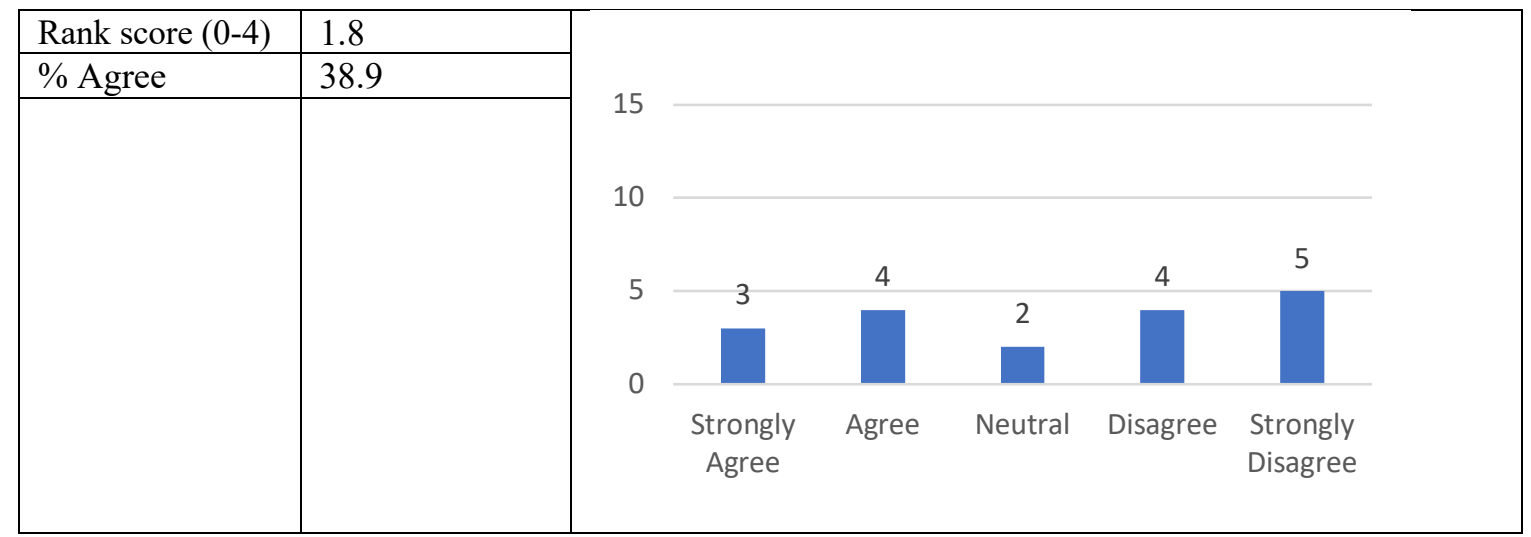


3. In order to determine leprosy endemicity, we should use the average indicator value of past three years. $(\mathrm{N}=18)$

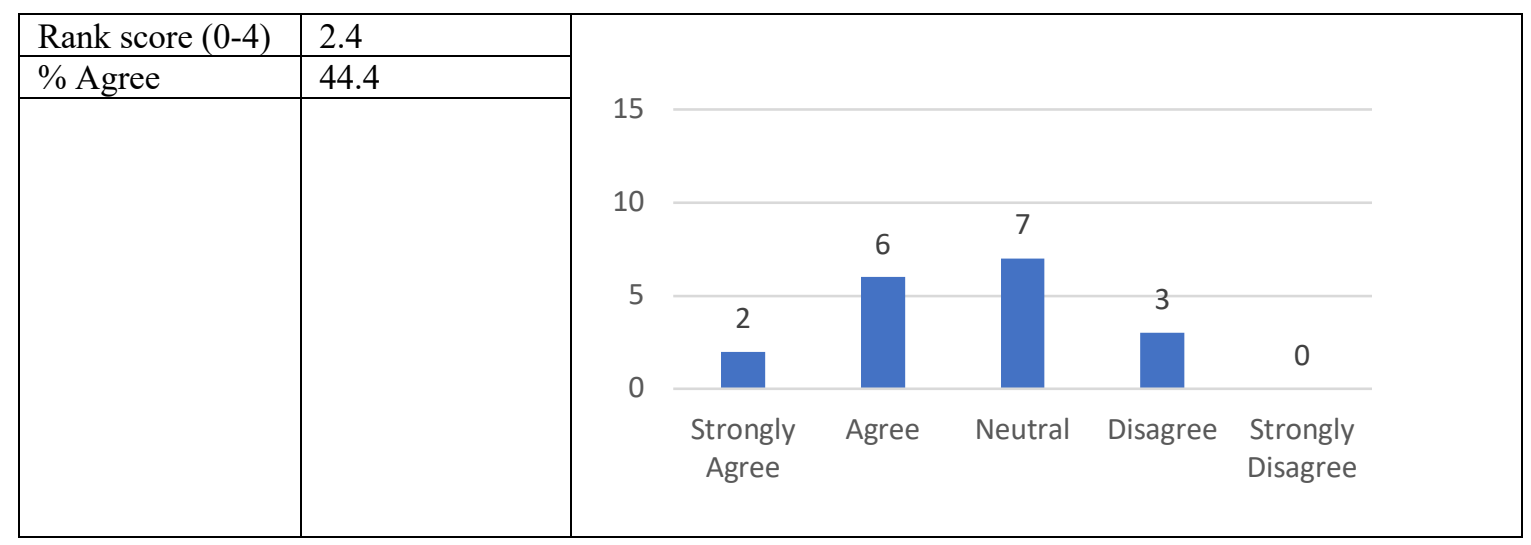

4. In order to determine leprosy endemicity, we should use the average indicator value of past five years. $(\mathrm{N}=18)$

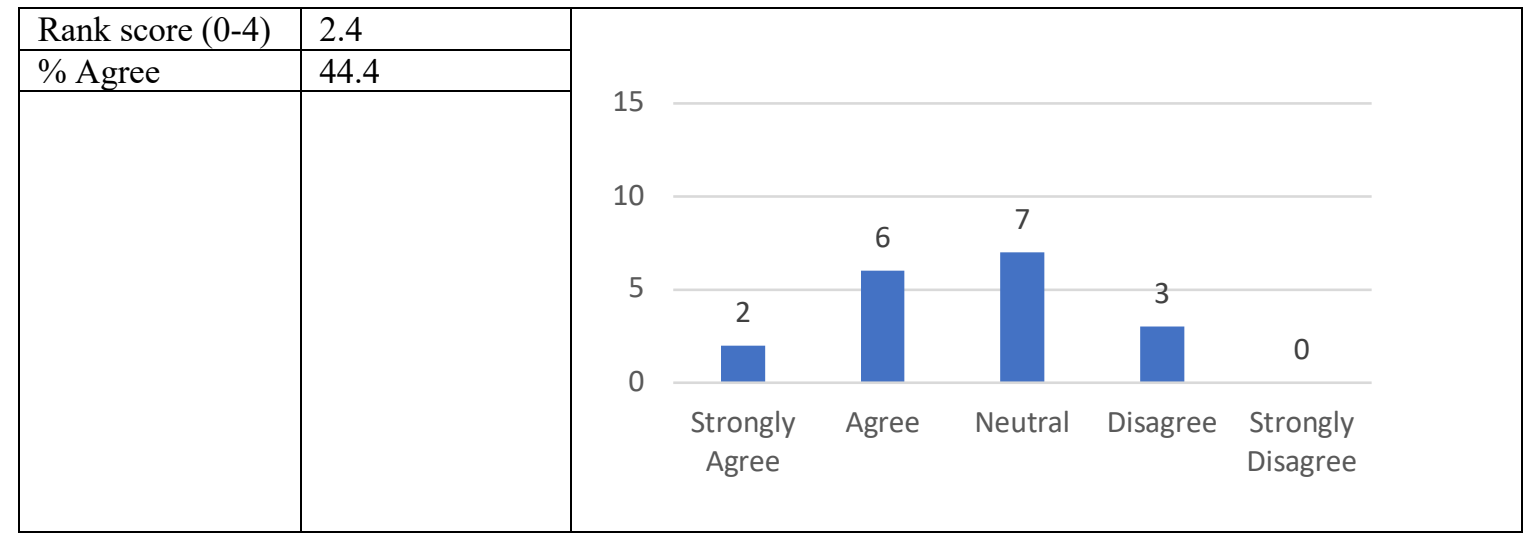

5. In order to determine leprosy endemicity, we should use the average indicator value of past ten years. $(\mathrm{N}=18)$

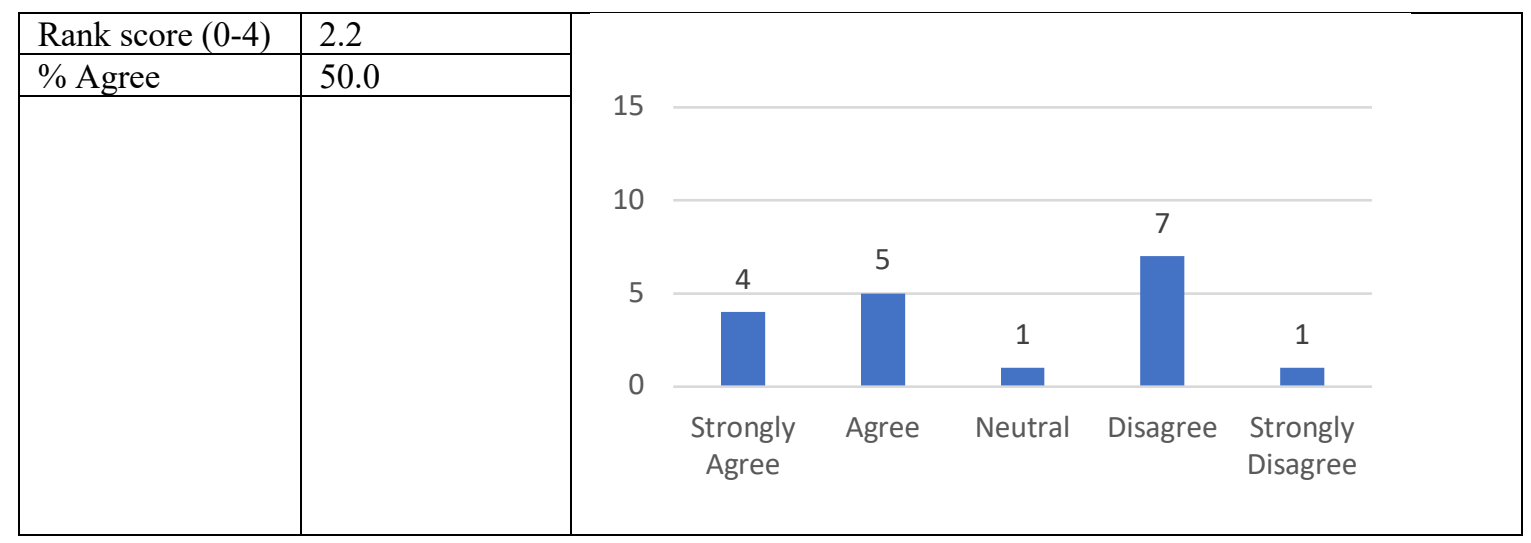


6. In order to determine leprosy endemicity, we should consider both the indicator value of a single year and the average indicator value of the past three/five/ten years. $(\mathrm{N}=18)$

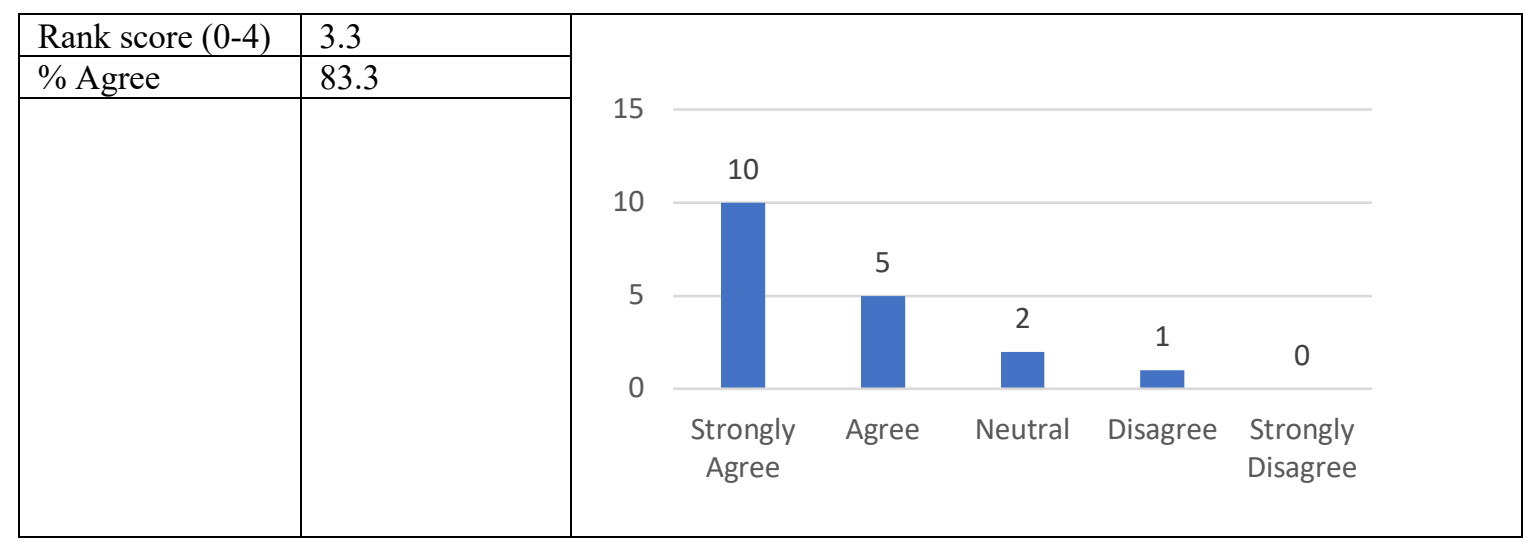

\section{Classification Levels of Endemicity}

7. Which of the following levels are relevant to categorize endemicity of leprosy at sub-national level? Please indicate your opinion against each of the mentioned levels. $(\mathrm{N}=15)$

Hyperendemic

\begin{tabular}{|c|c|c|c|c|c|}
\hline Rank score $(0-2)$ & 0.9 & & & & \\
\hline$\%$ Relevant & 60.0 & 14 & & & \\
\hline & & 12 & & & \\
\hline & & 10 & & & \\
\hline & & 8 & & & 6 \\
\hline & & 6 & 4 & 5 & \\
\hline & & & & & \\
\hline & & 0 & & & \\
\hline & & & Highly Relevant & Relevant & Not Relevant \\
\hline
\end{tabular}

High

\begin{tabular}{|c|c|c|c|c|c|}
\hline Rank score $(0-2)$ & 1.4 & \multirow[b]{2}{*}{14} & & & \\
\hline \multirow[t]{9}{*}{$\%$ Relevant } & 93.3 & & & & \\
\hline & & 12 & & & \\
\hline & & 10 & & & \\
\hline & & 8 & 7 & 7 & \\
\hline & & 6 & 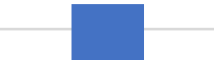 & & \\
\hline & & 4 & 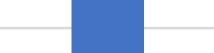 & & \\
\hline & & 2 & & & 1 \\
\hline & & 0 & & & $\square$ \\
\hline & & & Highly Relevant & Relevant & Not Relevant \\
\hline
\end{tabular}


Medium

\begin{tabular}{|c|c|c|c|c|c|}
\hline Rank score (0-2) & 1.1 & \multirow[b]{2}{*}{14} & & & \\
\hline$\%$ Relevant & 73.3 & & & & \\
\hline & & 12 & & & \\
\hline & & 10 & & & \\
\hline & & 8 & 6 & ᄃ & \\
\hline & & 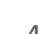 & & & 4 \\
\hline & & 2 & & & \\
\hline & & & Highly Relevant & Relevant & Not Relevant \\
\hline
\end{tabular}

Low

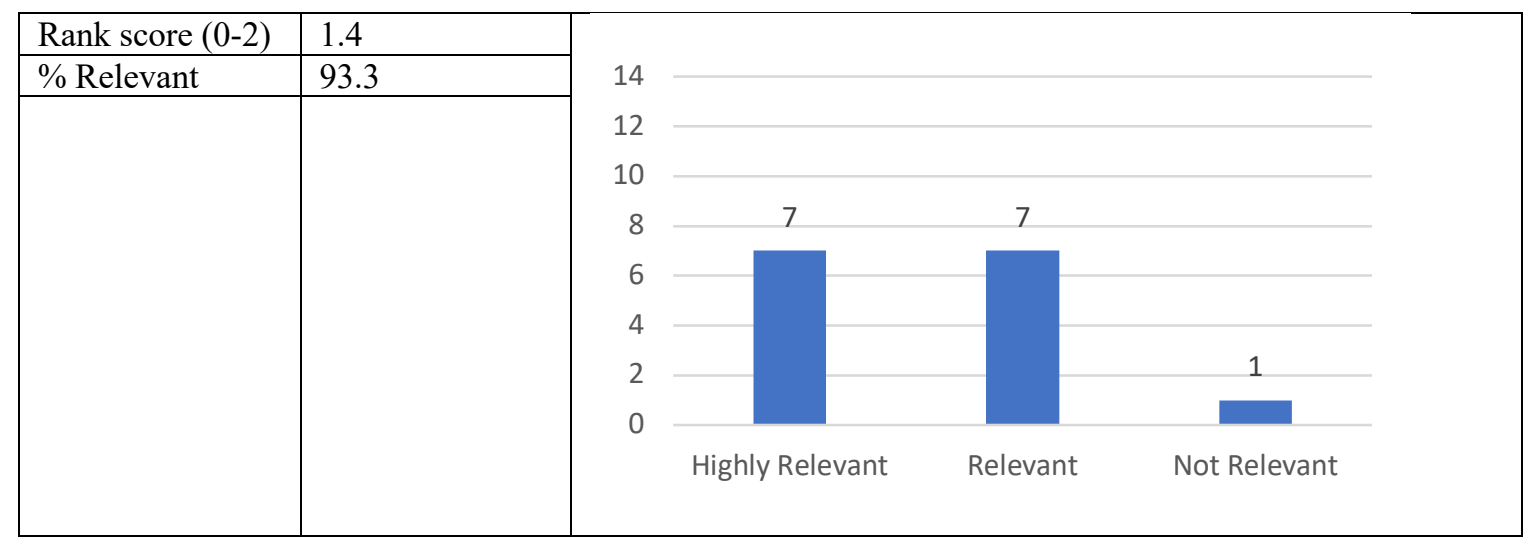

Non-endemic

\begin{tabular}{|c|c|c|c|c|c|}
\hline \multirow{2}{*}{$\begin{array}{l}\text { Rank score }(0-2) \\
\% \text { Relevant }\end{array}$} & 1.3 & \multirow[b]{2}{*}{14} & & & \\
\hline & 80.0 & & & & \\
\hline & & 12 & & & \\
\hline & & 10 & 8 & & \\
\hline & & & & & \\
\hline & & 4 & & & 3 \\
\hline & & 2 & & & \\
\hline & & & Highly Relevant & Relevant & Not Relevant \\
\hline
\end{tabular}


No specific level (i.e. endemic)

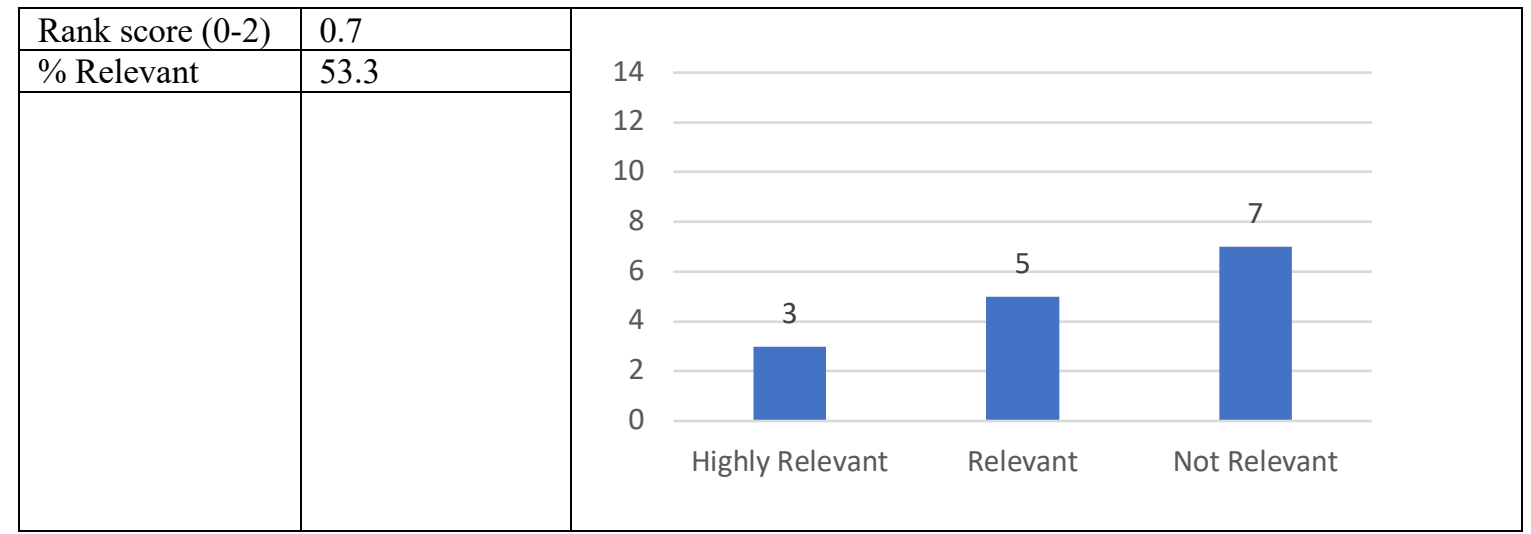

\section{Scoring Endemicity}

8. It is essential to score indicators based on threshold / cut-off values $(\mathrm{N}=14)$

\begin{tabular}{|c|c|c|c|c|c|c|c|}
\hline Rank score (0-4) & 2.7 & \multirow{2}{*}{14} & & & & & \\
\hline \multirow[t]{7}{*}{$\%$ Agree } & 57.1 & & & & & & \\
\hline & & 10 & & & & & \\
\hline & & 8 & & & & & \\
\hline & & 6 & & 5 & 5 & & \\
\hline & & 4 & & & & & \\
\hline & & 2 & & & & 1 & 0 \\
\hline & & & $\begin{array}{c}\text { Strongly } \\
\text { Agree }\end{array}$ & Agree & Neutral & Disagree & $\begin{array}{l}\text { Strongly } \\
\text { Disagree }\end{array}$ \\
\hline
\end{tabular}

9. There should be one standard for threshold / cut-off values to score indicators at subnational-level worldwide. $(\mathrm{N}=14)$

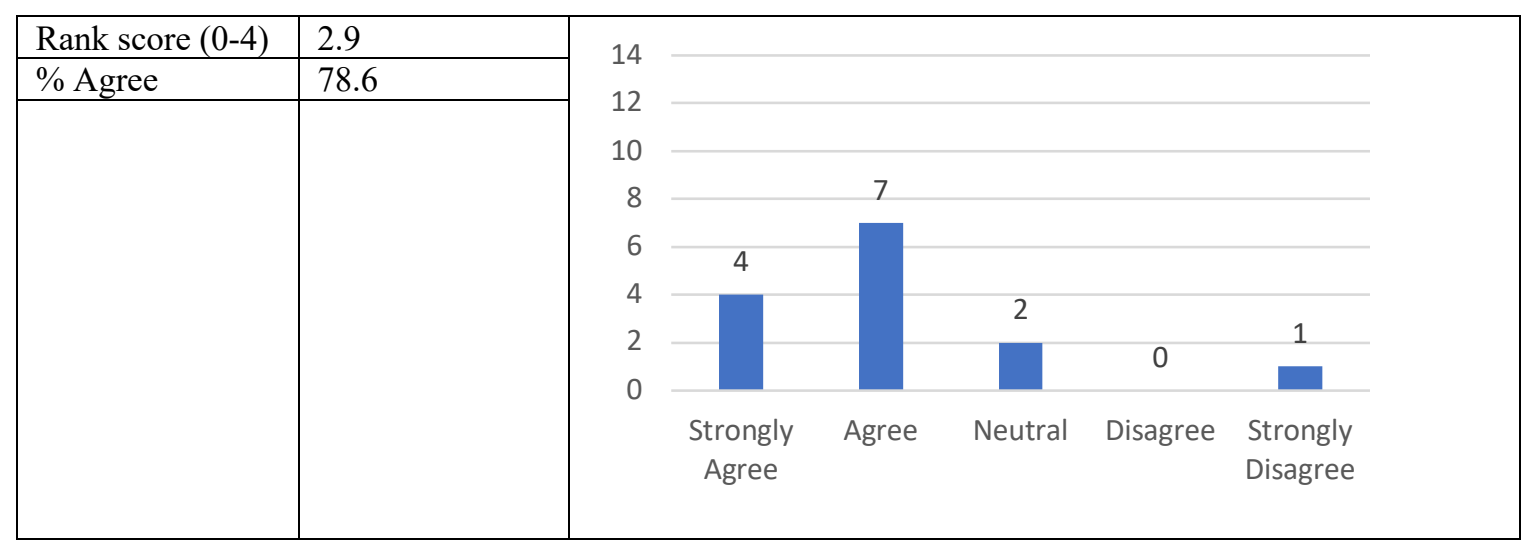


10. Which of the following scoring methods are relevant to determine endemicity of leprosy at subnational level? $(\mathrm{N}=14)$

Score of a single (most relevant) indicator (i.e. one overall classification level)

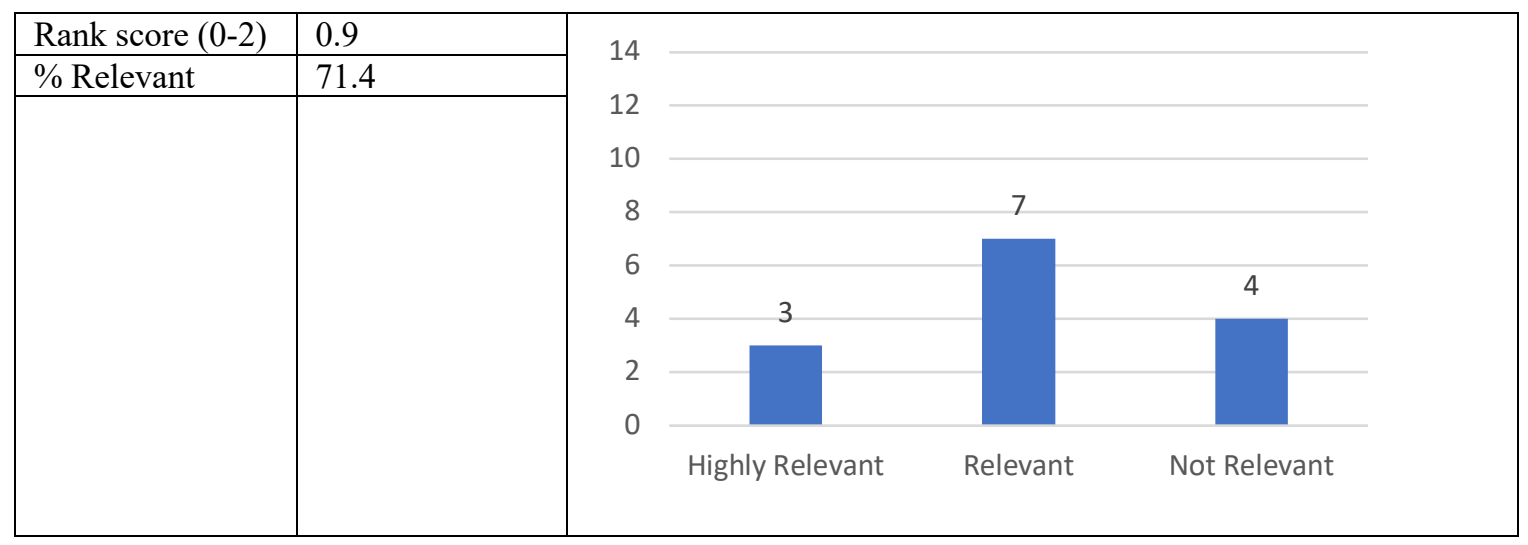

Composite score (i.e. one overall classification level based on multiple relevant indicators)

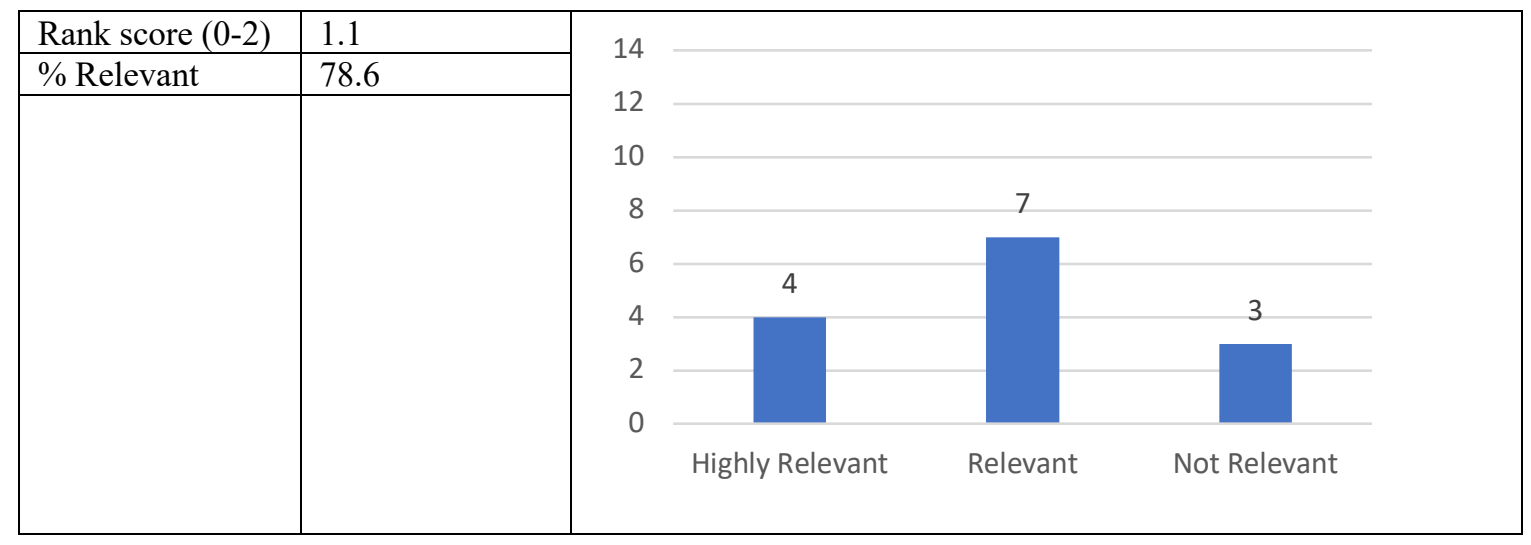

Score of multiple relevant indicators (i.e. multiple classification level: one for each indicator)

\begin{tabular}{|c|c|c|c|c|c|}
\hline Rank score $(0-2)$ & 1.2 & \multirow{2}{*}{14} & & & \\
\hline \multirow{8}{*}{$\%$ Relevant } & 92.9 & & & \multirow[b]{2}{*}{9} & \\
\hline & & 10 & & & \\
\hline & & 8 & & & \\
\hline & & 6 & 4 & & \\
\hline & & 4 & & & \\
\hline & & 2 & & & 1 \\
\hline & & 0 & & & 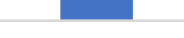 \\
\hline & & & Highly Relevant & Relevant & Not Relevant \\
\hline
\end{tabular}




\section{Indicators of Burden of Leprosy}

11. Which of the following indicators are relevant to classify burden of leprosy at sub-national level? Rank them in their order of importance: 1 being the most important and onward numbers being less important; two or more indicators may be ranked the same if they are equally important. Any indicator which you may consider non-relevant could be ranked as $N A$. $(\mathrm{N}=16)$

\begin{tabular}{|c|c|c|c|c|c|c|c|c|c|c|c|c|}
\hline \multirow[t]{2}{*}{ Indicator } & \multicolumn{10}{|c|}{ Ranking } & \multirow{2}{*}{$\begin{array}{l}\text { Rank } \\
\text { score } \\
(0-5) \\
\end{array}$} & \multirow{2}{*}{$\begin{array}{l}\% \\
\text { Rank } \\
\text { top-3 } \\
\end{array}$} \\
\hline & 1 & 2 & 3 & 4 & 5 & 6 & 7 & 8 & 9 & $10+$ & & \\
\hline New case detection (number and/or rate) & 3 & 2 & 1 & & & & & & & 3 & 1.4 & 33.3 \\
\hline Disability adjusted life year (DALY) & 2 & & 1 & 1 & & 2 & & & & 4 & 0.8 & 16.7 \\
\hline Prevalence (number and/or rate) & 2 & 1 & & 1 & & & 1 & & & 2 & 0.9 & 16.7 \\
\hline Prevalence of people with disabilities due to leprosy & 4 & & 1 & 1 & 1 & & & & & 1 & 1.4 & 27.8 \\
\hline New cases detected with G2D (number and/or rate) & 1 & 1 & 1 & 2 & 2 & & & & 1 & 0 & 1.0 & 16.7 \\
\hline Number of reactions, neuritis \& lasting disabilities & 1 & 3 & 1 & 2 & & & 1 & 1 & 1 & 1 & 1.3 & 27.8 \\
\hline $\begin{array}{l}\text { Number of children with disabilities caused by/ after } \\
\text { treatment }\end{array}$ & 1 & & 1 & 1 & & 3 & & 1 & & 2 & 0.6 & 11.1 \\
\hline Proportion of G2D cases among total new cases detected & 2 & & 1 & & 2 & 1 & & & & 1 & 0.8 & 16.7 \\
\hline Quality adjusted life year (QALY) & & 2 & & & & 1 & 1 & & 1 & 2 & 0.4 & 11.1 \\
\hline $\begin{array}{l}\text { Number requiring assistance (daily living, household } \\
\text { duties, work, etc.) }\end{array}$ & & 2 & 1 & & 2 & & & 2 & 1 & 1 & 0.7 & 16.7 \\
\hline Number of disabilities caused by/ after treatment & & 1 & 2 & & 1 & & 2 & & & 2 & 0.6 & 16.7 \\
\hline New cases detected among children (number and/or rate) & & 2 & & & & & & & & 2 & 0.4 & 11.1 \\
\hline Proportion of child cases among total new cases detected & & & 1 & & & & & & & 3 & 0.2 & 5.6 \\
\hline Proportion of MB cases among total new cases detected & & & 1 & 1 & & 1 & & & & 3 & 0.3 & 5.6 \\
\hline Number requiring surgery & & & & 1 & & & 2 & & 1 & 5 & 0.1 & 0.0 \\
\hline Proportion with psychological \& mental distress & & & & 1 & 1 & & 1 & 1 & 2 & 3 & 0.2 & 0.0 \\
\hline $\begin{array}{l}\text { Measures of social \& community impact (using stigma } \\
\text { scales) }\end{array}$ & & & & & 1 & 1 & 1 & 2 & 1 & 2 & 0.1 & 0.0 \\
\hline Cost of treatment \& rehabilitation & & & & & & 1 & & 2 & & 5 & 0.0 & 0.0 \\
\hline
\end{tabular}

\section{Usage of Indicators to Determine Burden of Leprosy}

12. In order to determine leprosy burden, we should use the indicator value of a single year. $(\mathrm{N}=16)$

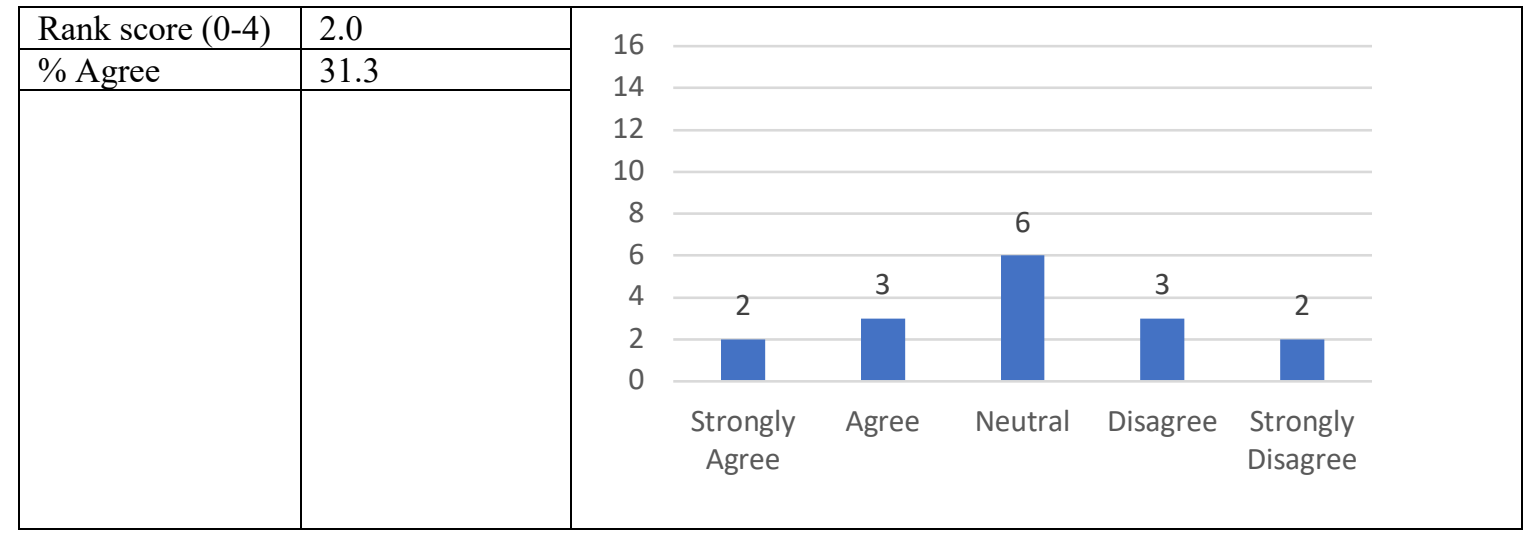


13. In order to determine leprosy burden, we should use the average indicator value of past years (e.g. thee or five years). $(\mathrm{N}=16)$

\begin{tabular}{|c|c|c|c|c|c|c|c|}
\hline Rank score (0-4) & 3.1 & \multirow{2}{*}{16} & & & & & \\
\hline \multirow[t]{8}{*}{$\%$ Agree } & 87.5 & & & & & & \\
\hline & & 12 & & 11 & & & \\
\hline & & 10 & & 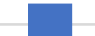 & & & \\
\hline & & 8 & & & & & \\
\hline & & 6 & & & & & \\
\hline & & 4 & 3 & & 2 & & \\
\hline & & 2 & & & & 0 & 0 \\
\hline & & & $\begin{array}{c}\text { Strongly } \\
\text { Agree }\end{array}$ & Agree & Neutral & Disagree & $\begin{array}{l}\text { Strongly } \\
\text { Disagree }\end{array}$ \\
\hline
\end{tabular}

14. In order to determine leprosy burden, we should consider both the indicator value of a single year and the average indicator value of the past three or five years. Burden is determined by the highest of the two. $(\mathrm{N}=16)$

\begin{tabular}{|c|c|c|c|c|c|c|c|}
\hline Rank score (0-4) & $\frac{3.1}{750}$ & \multirow{8}{*}{$\begin{array}{r}16 \\
14 \\
12 \\
10 \\
8 \\
6 \\
4 \\
2 \\
0\end{array}$} & & & & & \\
\hline$\%$ Agree & 75.0 & & & & & & \\
\hline & & & & & & & \\
\hline & & & 7 & & & & \\
\hline & & & & 5 & & & \\
\hline & & & & & 3 & & \\
\hline & & & & & & 1 & 0 \\
\hline & & & $\begin{array}{l}\text { Strongly } \\
\text { Agree }\end{array}$ & Agree & Neutral & Disagree & $\begin{array}{l}\text { Strongly } \\
\text { Disagree }\end{array}$ \\
\hline
\end{tabular}

\section{Classification Levels of Burden of Leprosy}

15. Which of the following levels are relevant to categorize burden of leprosy at sub-national level? Please indicate your opinion against each of the mentioned levels. $(\mathrm{N}=16)$

Very High

\begin{tabular}{|c|c|c|c|c|c|}
\hline Rank score (0-2) & 1.1 & \multirow{2}{*}{16} & & & \\
\hline \multirow[t]{7}{*}{ \% Relevant } & 68.8 & & & & \\
\hline & & 12 & & & \\
\hline & & 10 & & & \\
\hline & & 8 & 6 & & \\
\hline & & 1 & & & \\
\hline & & 2 & 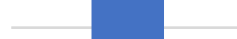 & & \\
\hline & & & Highly Relevant & Relevant & Not Relevant \\
\hline
\end{tabular}


High

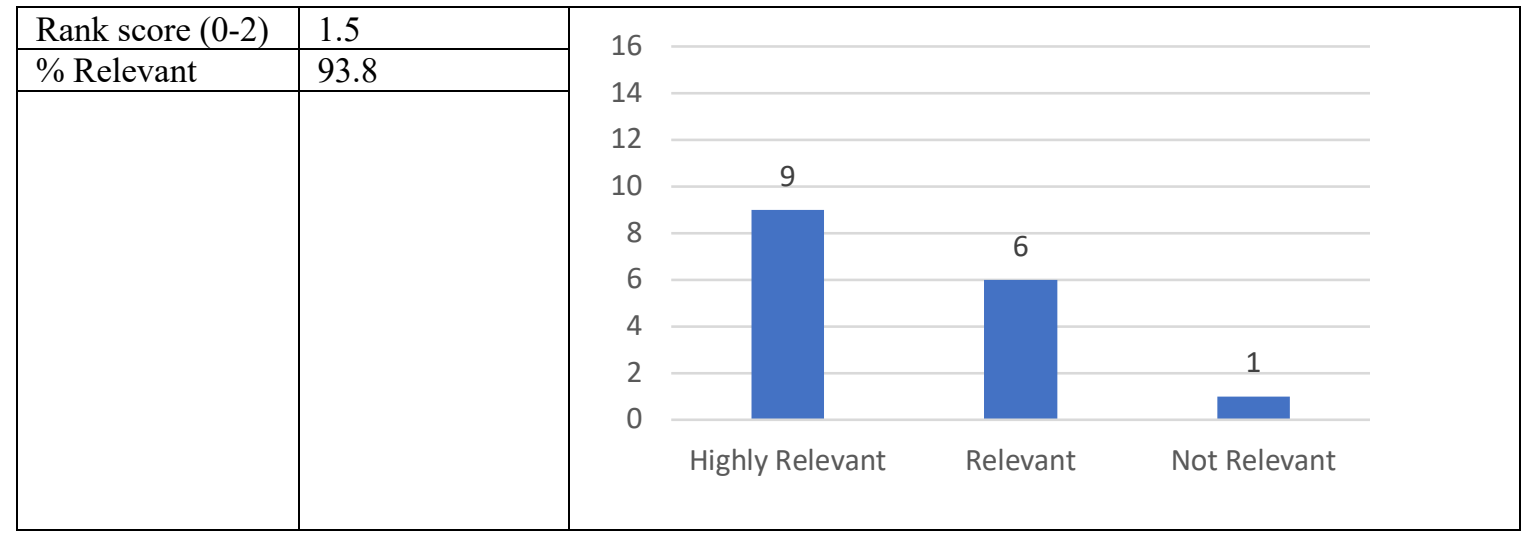

\section{Medium}

\begin{tabular}{|c|c|c|c|c|c|}
\hline Rank score (0-2) & 0.9 & \multirow{2}{*}{16} & & & \\
\hline \multirow[t]{8}{*}{$\%$ Relevant } & 62.5 & & & & \\
\hline & & 12 & & & \\
\hline & & 10 & & & \\
\hline & & 8 & & & 6 \\
\hline & & 6 & 5 & 5 & \\
\hline & & 4 & 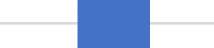 & & \\
\hline & & 2 & 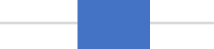 & & \\
\hline & & & Highly Relevant & Relevant & Not Relevant \\
\hline
\end{tabular}

\section{Low}

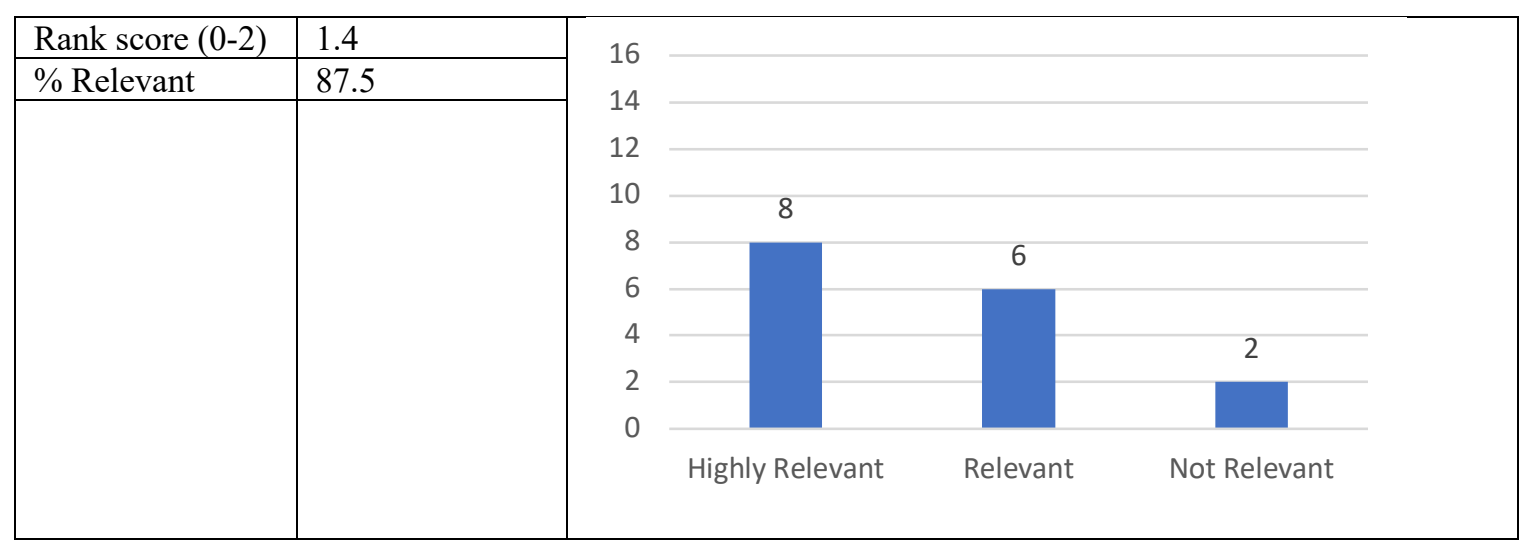


No burden

\begin{tabular}{|c|c|c|c|c|c|}
\hline & 1.3 & \multirow{2}{*}{$\begin{array}{l}16 \\
14\end{array}$} & & & \\
\hline & 75.0 & & & & \\
\hline \multirow{7}{*}{ \% Relevant } & & 12 & & & \\
\hline & & 10 & 8 & & \\
\hline & & 8 & & & \\
\hline & & 6 & & 4 & 4 \\
\hline & & 4 & & & \\
\hline & & 2 & 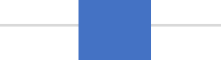 & & \\
\hline & & & Highly Relevant & Relevant & Not Relevant \\
\hline
\end{tabular}

\section{Scoring Burden of Leprosy}

16. It is essential to score indicators based on threshold / cut-off values. $(\mathrm{N}=15)$

\begin{tabular}{|c|c|c|c|c|c|c|c|}
\hline Rank score (0-4) & 2.8 & \multirow{3}{*}{$\begin{array}{l}14 \\
12\end{array}$} & & & & & \\
\hline \multirow[t]{7}{*}{$\%$ Agree } & 73.3 & & & & & & \\
\hline & & & & & & & \\
\hline & & 10 & & 8 & & & \\
\hline & & $\begin{array}{l}8 \\
6\end{array}$ & & & & & \\
\hline & & 4 & & & & 2 & \\
\hline & & 2 & & & & & 0 \\
\hline & & & $\begin{array}{l}\text { Strongly } \\
\text { Agree }\end{array}$ & Agree & Neutral & Disagree & $\begin{array}{l}\text { Strongly } \\
\text { Disagree }\end{array}$ \\
\hline
\end{tabular}

17. There should be one standard for threshold / cut-off values to score indicators at subnational-level worldwide. $(\mathrm{N}=15)$

\begin{tabular}{|c|c|c|c|c|c|c|c|}
\hline \multirow{2}{*}{$\begin{array}{l}\text { Rank score }(0-4) \\
\% \text { Agree }\end{array}$} & $\begin{array}{l}2.3 \\
600\end{array}$ & \multirow{3}{*}{$\begin{array}{l}14 \\
12\end{array}$} & & & & & \\
\hline & 60.0 & & & & & & \\
\hline & & & & & & & \\
\hline & & 10 & & 8 & & & \\
\hline & & 8 & & & & & \\
\hline & & 4 & & & 2 & 3 & \\
\hline & & 2 & 1 & & 2 & - & 1 \\
\hline & & 0 & $=$ & & & & 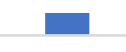 \\
\hline & & & $\begin{array}{c}\text { Strongly } \\
\text { Agree }\end{array}$ & Agree & Neutral & Disagree & $\begin{array}{l}\text { Strongly } \\
\text { Disagree }\end{array}$ \\
\hline
\end{tabular}


18. Which of the following scoring methods are relevant to determine burden of leprosy at subnational level? $(\mathrm{N}=15)$

Score of a single (most relevant) indicator (i.e. one overall classification level)

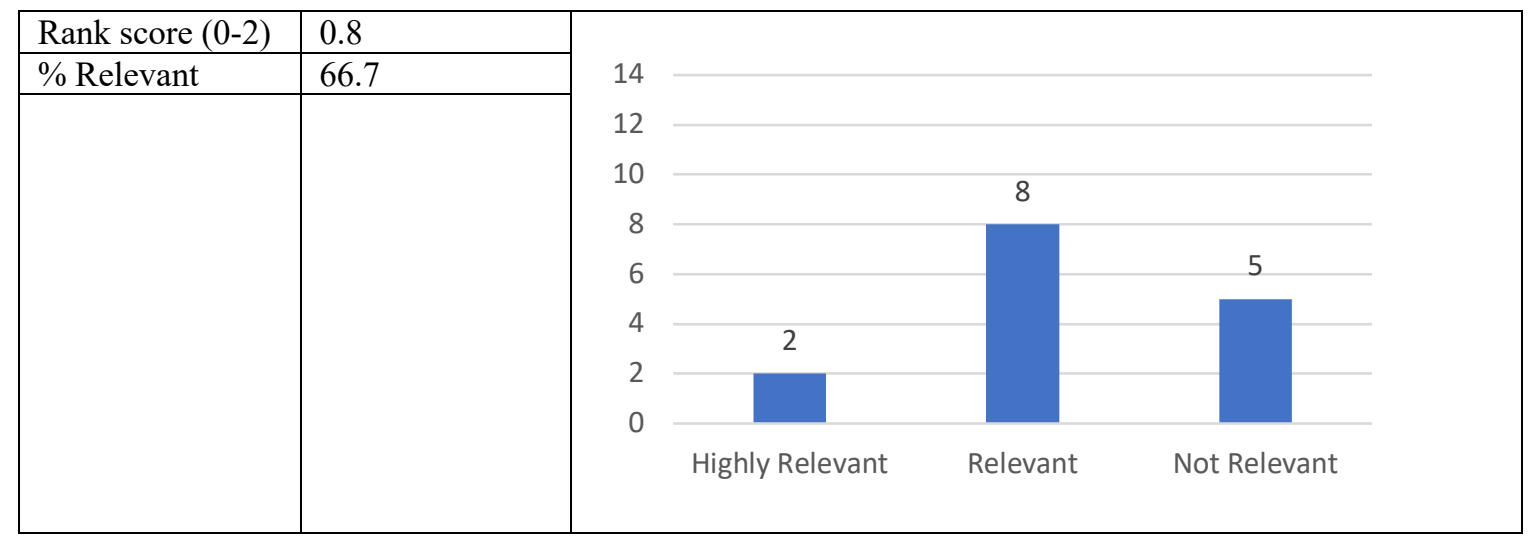

Composite score (i.e. one overall classification level based on multiple relevant indicators)

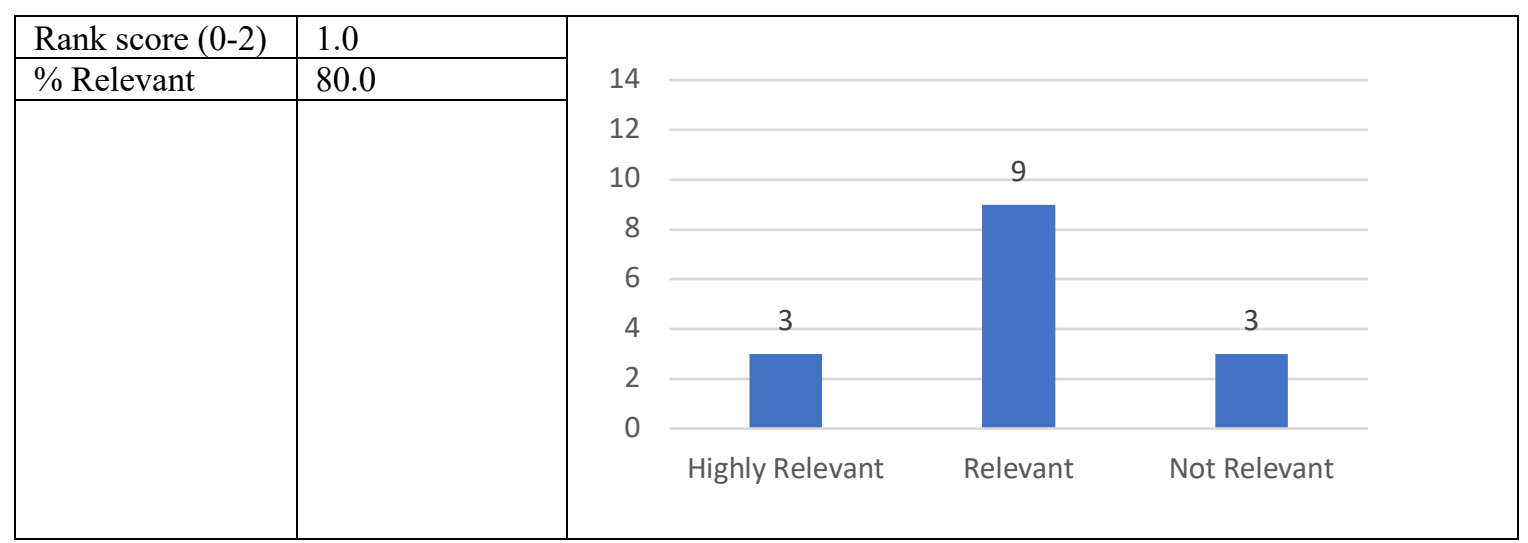

Score of multiple relevant indicators (i.e. multiple classification level: one for each indicator)

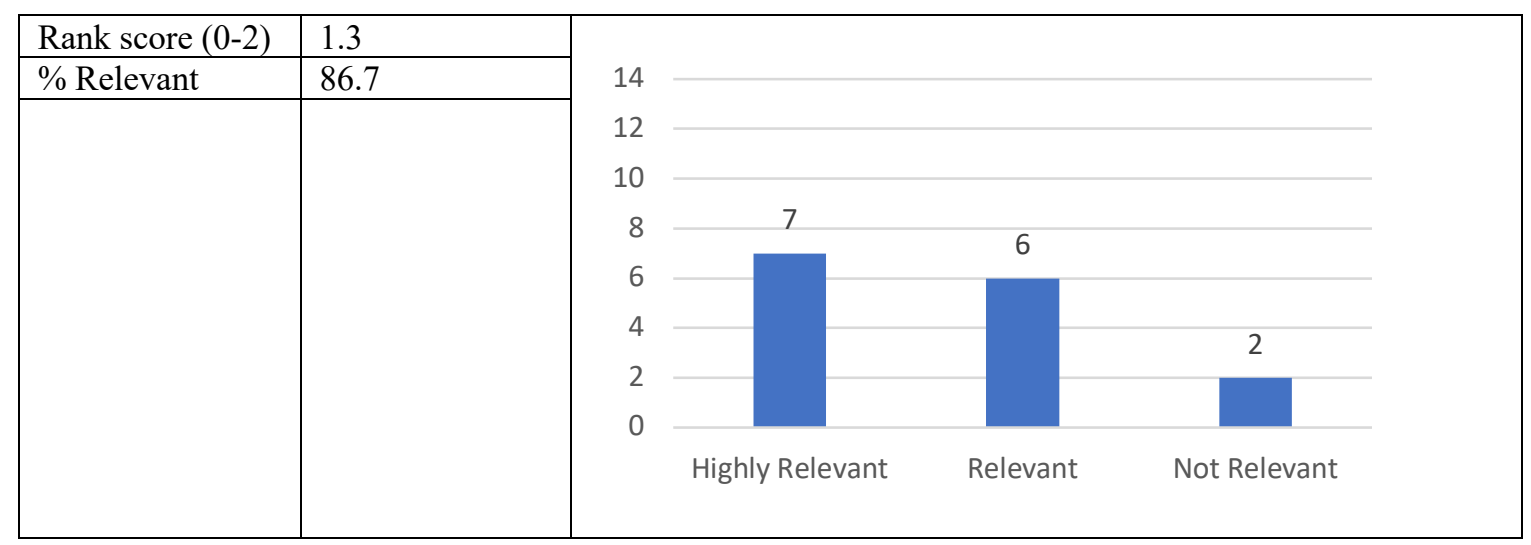

